\title{
Unsteady dynamics and organized structures from DNS over an idealized building canopy
}

\author{
Omduth Coceal, ${ }^{a *}$ Adrian Dobre ${ }^{\mathrm{b}}$ and T. G. Thomas ${ }^{\mathrm{c}}$ \\ a Department of Meteorology, University of Reading, U.K. \\ b National Institute for Aerospace Research "Elie Carafoli", Bucharest, Romania \\ c School of Engineering Sciences, University of Southampton, U.K.
}

\begin{abstract}
:
A numerical study is performed to elucidate the dominant turbulent processes that occur in urban areas. Comprehensive data from direct numerical simulations (DNS) over idealized three-dimensional arrays of buildings are analysed to study the unsteady and organized aspects of the turbulent flow. The accuracy of the DNS is evaluated by comparing turbulence statistics with a high quality wind-tunnel dataset. The simulation results are studied using flow visualization as well as statistical methods including quadrant analysis, space-time two-point correlations and conditional averaging. Three regimes of the flow are identified. First, the rough wall flow above the buildings has turbulent organized structures that resemble the 'hairpin vortices' and 'low momentum regions' that are well known to occur in the turbulent boundary layer over smooth walls. These hairpin-like vortices contribute dominantly to vertical momentum transport. Secondly, shear layers develop over the tops of the buildings and shed structures that may sometimes impinge upon downstream buildings and drive a robust recirculation within the building canopy. These unsteady canopy-top shear layers and their interaction with the larger eddies above provide important mechanisms for coupling with the flow within the canopy. Thirdly, the flow within the building canopy is the result of complicated interactions between the above and eddies shed off the vertical edges of the buildings, and their distortion caused by impacting buildings. Mean flow patterns around the buildings are important and lead to significant dispersive stresses. Implications for scalar transport and dispersion are briefly discussed. Copyright (C) 2007 Royal Meteorological Society
\end{abstract}

KEY WORDS DNS; coherent structures; turbulent organized structures; urban canopy

Received 5 October 2006; Revised 20 March 2007; Accepted 23 March 2007

\section{INTRODUCTION}

Urban turbulence is a complex subject for two distinct reasons. The first is the heterogeneity of urban areas, which usually consist of buildings of different shapes, sizes and layouts. The second reason is the inherent complexity of turbulent flows, even over relatively simple building configurations. Numerous field campaigns have been performed in different cities, but the interpretation of the measured data is often not straightforward owing to a lack of basic understanding of turbulent flow characteristics over groups of buildings. Moreover, recent interest in urban air quality has made it important to understand turbulent processes at the street level and on correspondingly small spatial and time scales. It seems likely that applications that depend on the flow dynamics on short timescales, such as the dispersion of pollutants, may require a much more detailed understanding of the unsteady flow than is available at present.

\footnotetext{
* Correspondence to: Omduth Coceal, Department of Meteorology, University of Reading, P.O. Box 243, Reading, RG6 6BB, U.K. E-mail: o.coceal@reading.ac.uk
}

A good amount of progress has been made in recent years in studying the mean aspects of the flow at the street scale and neighbourhood scale (see for e.g. the review by Britter and Hanna, 2003), but little is currently known about the unsteady flow dynamics. It is now believed that unsteady effects due to organized or coherent structures in urban turbulence must be at least as important, in an order of magnitude sense, as the mean flow (Oikawa and Meng, 1995; Feigenwinter and Vogt, 2005). Yet we still do not have a conceptual model of the unsteady flow, let alone a detailed quantitative understanding that might lend itself to future parameterizations.

The aim of this paper is to begin to develop such a conceptual model. This involves identifying features and processes that may be important, and is best achieved by considering relatively idealized building configurations. It is then possible to focus on the dynamics of the turbulence itself, rather than the complicating physical heterogeneity of the urban environment. Such a conceptual understanding could form the basis of future parameterizations that could be incorporated into simpler predictive models such as urban canopy models (Coceal and Belcher, 2004, 2005). 
A good starting point is to examine existing canonical flows that might potentially bear some resemblance with the present problem, and then to ask how they differ. In terms of turbulence dynamics, there are at least two extensively studied flows in the literature that one could compare with, and they lead to different paradigms. First, one could think of the flow above the buildings as a rough wall boundary layer (Raupach et al., 1991; Jimenez, 2004). Raupach et al. (1991) suggested that well above the roughness the turbulence structure should have universal characteristics independent of the nature of the rough surface, and should in fact resemble a smooth wall boundary layer, but this view is not universally accepted.

Secondly, the aggregate of buildings could be considered as a porous medium, or urban canopy, and compared with a vegetation canopy. Raupach et al. (1996) proposed a model of canopy turbulence based on an analogy with the inviscid instability of a plane mixing layer. It is frequently thought that many characteristics of urban turbulence could also be explained within a similar framework. Observations of organized motions in the urban boundary layer have been interpreted as supporting this view, at least partially (Roth, 2000; Christen, 2005). However, it is clear that mechanisms other than those present in vegetation canopies are important, such as vortex shedding by the sharp edges of the buildings. The apparent similarities between urban and plant canopy turbulence have been analysed in terms of integral statistics and spectra, but more detailed study is necessary to look at actual organized structures and the mechanisms of their production.

Kanda et al. (2004) and Kanda (2006) have recently performed large-eddy simulations over cube arrays and demonstrated important differences between the turbulence structure over urban-like roughness compared to vegetation canopies. Kanda et al. (2004) showed that the scales of structures over so-called 'd-type' cube arrays (such as aligned arrays) do not match those predicted by the mixing-layer analogy, but Kanda (2006) showed evidence that some mixing-layer characteristics may be present over so-called 'k-type' arrays (such as staggered arrays). Moreover, these authors demonstrated the existence of low speed streaks and streamwise vortices, which are well known to exist in flat wall boundary layers.

The work of Kanda et al. (2004) and Kanda (2006) has highlighted the importance of documenting the detailed spatial structure of the organized motions, instead of relying on single-point statistics such as quadrant analysis, which can yield inconclusive and sometimes conflicting results (Kanda, 2006). These authors used flow visualization to document the coherent structures, but ideally a combination of tools is needed to study different aspects of the coherent structures as well as their statistical properties. Further questions that need to be clarified include the nature of turbulent structures within the building canopy and the interaction with the flow above. The strong three-dimensional nature of turbulence requires comprehensive data in 3D space at high enough spatial and time resolution. Such information is difficult to obtain experimentally, but is readily generated by numerical simulations. Coceal et al. (2006) recently reported detailed novel results on the mean flow and turbulent statistics over arrays of urban-like cubical obstacles. In the present paper we exploit the capabilities of these direct numerical simulations (DNS) in providing information about the unsteady flow as well as the mean flow. We focus on the organized aspects of the unsteady flow.

The paper is organized as follows. The second section (numerical simulations) outlines the numerical methodology, gives details of the runs and shows a comparison of turbulence statistics with a wind tunnel dataset. The third section (results on organized aspects of the unsteady flow) then shows the results of flow visualization and statistical analysis of unsteady flow and organized motions above and within the building array. The fourth section concludes with a summary followed by a discussion of remaining questions.

\section{NUMERICAL SIMULATIONS}

\section{Numerical method and geometry}

DNS are performed by discretizing the Navier-Stokes equations using second-order finite differences in space and time. The code is parallelized in a highly efficient manner using MPI, and a flexible multi-block mapping strategy is developed to deal with the flow domain containing the complex geometry. The Poisson equation for pressure is solved by a multigrid method. Further details of numerical methods are given in Coceal et al. (2006) and Yao et al. (2001).

The simulated geometry consists of a regular array of cubes (height $h$ ) placed in a staggered arrangement as shown in Figure 1. The separation between adjacent cubes is $h$ in the lateral direction and $3 h$ in the streamwise direction, so that the packing density, $\lambda_{\mathrm{f}}=$ $\lambda_{\mathrm{p}}=0.25$ (with $\lambda_{\mathrm{f}}$ and $\lambda_{\mathrm{p}}$ as defined in Grimmond and Oke, 1999). The notational convention adopted here is that $(x, y, z)$ denote the coordinates, and $(u, v, w)$ denote the velocity components, in the streamwise, lateral and vertical directions respectively. The computational domain has streamwise, lateral and vertical dimensions of $16 h, 12 h$ and $8 h$ respectively. This choice of domain size was guided by the results of wind tunnel measurements made by Castro et al. (2006) over the same geometry. These authors deduced integral lengthscales in the streamwise, lateral and vertical directions of $L_{\mathrm{x}}=$ $3 h, L_{\mathrm{y}}=h$ and $L_{\mathrm{z}}=h$ in the inertial sublayer. The present dimensions of the domain are chosen to be much larger than these integral length scales. The domain height $H=8 h$ is comparable to the boundary layer height $\delta=7.5 \mathrm{~h}$ in the wind tunnel experiment of Cheng and Castro (2002) and Castro et al. (2006).

Periodic boundary conditions are imposed in the streamwise and lateral directions, a free slip boundary 


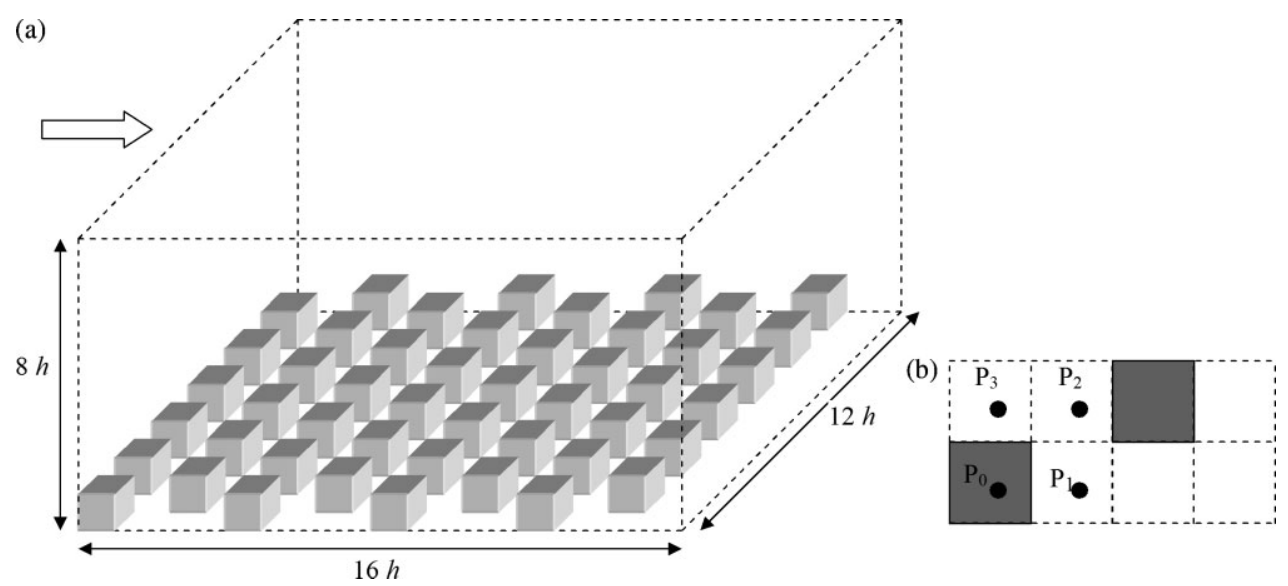

Figure 1. (a) Computational domain for the DNS. (b) Plan view of a 'repeating unit'.

condition is imposed at the top of the domain, and noslip is imposed on the bottom wall and on all obstacle surfaces. The flow is driven by a height-independent pressure gradient of magnitude $u_{\tau}{ }^{2} / H$, where $u_{\tau}$ is the friction velocity based on the total stress on the rough surface. The Reynolds number is $R e=5800$ based on the velocity at the top of the domain and the cube height, and the corresponding roughness Reynolds number is $R e_{\tau}=u_{\tau} h / v=500$. This Reynolds number is in the fully rough regime (Raupach et al., 1991). An alternative definition of roughness Reynolds number $\left(R e^{*}\right)$ $=u_{\tau} z_{0} / v$ is sometimes used in the literature (Snyder and Castro, 2002), where $z_{0}$ is the roughness length. Here, the value of $R e^{*}$ is 22.5. A Reynolds number of $R e=5800$ is very small compared to typical Reynolds numbers encountered in real atmospheric flows, and the range of scales simulated here is correspondingly more limited. However, Xie and Castro (2006) have recently performed large eddy simulations (LES) over the same array of cubical buildings at Reynolds numbers of up to $R e=5 \times 10^{6}$, and they concluded that Reynolds number dependency (if any) is very weak for these types of flows. This is in contrast to flows over smooth surfaces or around isolated bodies, and is because the turbulence is dominated by eddy shedding from the sharp edges of urban-like obstacles. Statistics were collected over a duration of $100 T$ after an initial spin up time of $100 T$, where $T=h / u_{\tau}$ is the eddy turnover time for the largest eddies shed by the cubes. There are 24 'repeating units' in the array (48 if an additional reflectional symmetry is taken into account; Figure 1). Statistics can therefore be ensemble-averaged over these repeating units to increase the effective averaging time. The adequacy of the averaging time is reflected in smooth profiles of total stress as well as negligibly small dispersive stress above the canopy (Coceal et al., 2006). The simulation took a total cpu time of 384 hours on each of 124 processors, or an equivalent of about 48000 single processor cpu hours, on an SGI Altix 3700 supercomputer.

A uniform Cartesian grid was used with grid size $\Delta=$ $h / 32$, with the first gridpoint from solid surfaces being at a distance of $\Delta / 2$. This grid resolution is sufficient to capture most of the dissipation at the simulated Reynolds number (Coceal et al., 2006). Moreover, as noted above, the Reynolds number dependence for this type of flow is weak (Xie and Castro, 2006). The adequacy of the grid was confirmed by a close comparison of the statistics with those for a test run at double the resolution but on a smaller domain of $4 h \times 4 h \times 4 h$.

\section{Comparison with wind tunnel data}

Figure 2 demonstrates the good agreement obtained with the wind-tunnel data of Cheng and Castro (2002) and Castro et al. (2006). Further comparisons are presented in Coceal et al. (2006). Figure 2(a) shows the average of mean velocity profile measurements made at four horizontal positions $\mathrm{P}_{0}, \mathrm{P}_{1}, \mathrm{P}_{2}$ and $\mathrm{P}_{3}$ which are respectively located on top of, behind, in front of a cube and in the gap between two adjacent cubes (Figure 1(b)). Note the inflected shape of the profile at the canopy top.

Cheng and Castro (2002) measured the mean pressure on the front and back faces of a cube using pressure tappings. These measurements are used to compute a profile of the drag force exerted by the cubes. The pressure profile shown in Figure 2(b) is obtained by laterally integrating across the cube the pressure difference between corresponding points on the front and back faces, and normalising by the mean pressure difference. The result is compared with computations from the DNS in Figure 2(b), yielding excellent agreement.

Profiles of shear stress and streamwise turbulence intensity at the locations $\mathrm{P}_{1}$ and $\mathrm{P}_{2}$ are shown in Figure 2(c) and (d) and compare well with the wind tunnel measurements. There are relatively small differences between the computed streamwise turbulence intensity within the array, with the computed values being slightly lower.

The good comparisons in these long time-integrated first and second order statistics are further evidence of the accuracy of the simulations in capturing the essential dynamics of this highly turbulent flow. 
(6)
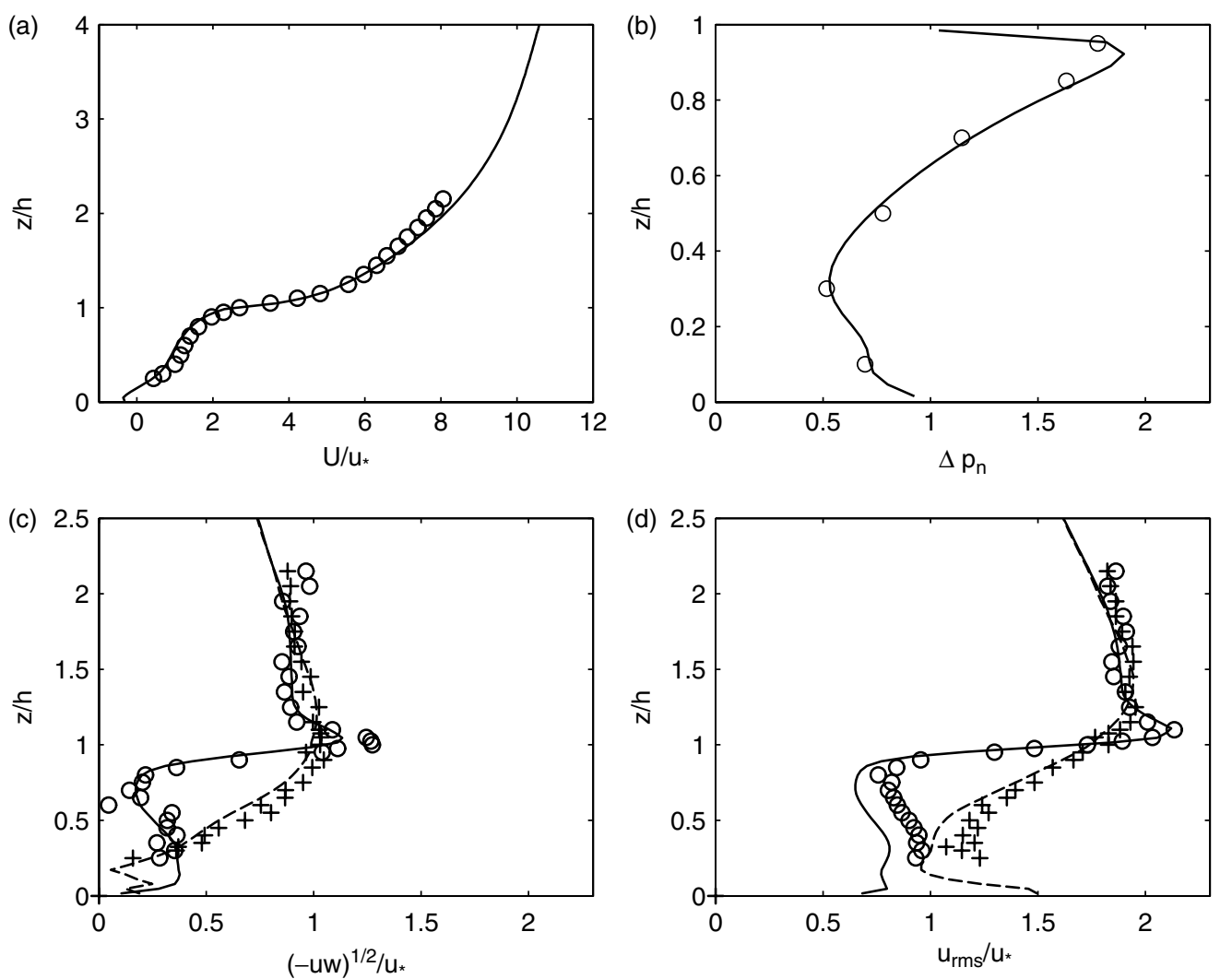

Figure 2. Comparison of DNS (lines) with wind tunnel data (symbols) of (a) mean velocity profile obtained as an average of profiles above points $\mathrm{P}_{0}, \mathrm{P}_{1}, \mathrm{P}_{2}$, and $\mathrm{P}_{3}$ (b) profile of laterally-integrated normalized pressure difference $\Delta \mathrm{p}_{\mathrm{n}}$ between the front and back faces of a building, (c) and (d): profiles of shear stress and $\mathrm{u}_{\mathrm{rms}}$ behind cube, $\mathrm{P}_{1}$ (solid lines and circles), and in front of cube, $\mathrm{P}_{2}$ (dashed lines and plus symbols).

\section{RESULTS ON ORGANIZED ASPECTS OF THE UNSTEADY FLOW}

We now turn our attention to the organized aspects of the unsteady flow, and show results using a combination of flow visualization and statistical tools such as two-point correlations, quadrant analysis and conditional averaging.

For convenience, results are reported in three separate sections - the above canopy flow, the canopy-top shear layer, and the canopy layer - but the coupling between these three regions is also explored along the way.

\section{Coherent structures above the buildings}

Flow visualization of streamwise velocity contours in instantaneous snapshots readily reveals the presence of large-scale structures described in the literature as low momentum regions (LMRs; Adrian et al., 2000; Tomkins and Adrian, 2003) or low speed streaks (Kanda et al., 2004; Kanda, 2006). These LMRs are present in most snapshots, showing that they are persistent, coherent features. On long timescales they may move around laterally. They are also intermittently disrupted on timescales of order 50-100 $T$ (corresponding to tens of minutes in full scale), and this appears to be related to intermittent outflows from the building canopy ( $c f$. Grass, 1971).

Figure 3 shows examples of LMRs visualized in horizontal planes at two different heights of $z=1.5 \mathrm{~h}$ and $z=2 h$. There are fewer streaks at higher levels but they are thicker on average. Tomkins and Adrian (2003) performed PIV measurements over a smooth wall in a wind tunnel, and they showed that the characteristic length and width of the streaks in the log layer increases linearly with distance above the surface. Detailed statistical analysis of our results (not shown) indicates that essentially the same results hold over the present, very rough surface.

The significance of low speed streaks is that they are associated with vortical structures that are believed to contribute dominantly to turbulent transport of momentum. According to the parent-offspring mechanism proposed by Adrian et al. (2000), LMRs are the result of coherent groups, or packets, of so-called hairpin (horseshoe) vortices. The mechanism by which these hairpin packets and the corresponding streaks form was investigated numerically by Zhou et al. (1999) and may be summarized as follows. Given a flow with mean shear as in any wall-bounded flow, a strong ejection event from an unspecified source kinks spanwise vortex lines that are then rotated and stretched by the mean shear. This results in an inclined primary vortex that resembles a horseshoe or hairpin shape. Because of the sense of rotation of the primary vortex, it produces a region of strong ejections in between the legs and weaker sweeps on the sides. The strong ejection in turn interacts with ambient spanwise vorticity upstream and spawns a further, secondary, hairpin. This process repeats a few times so that there are a number of hairpin vortices roughly aligned in 

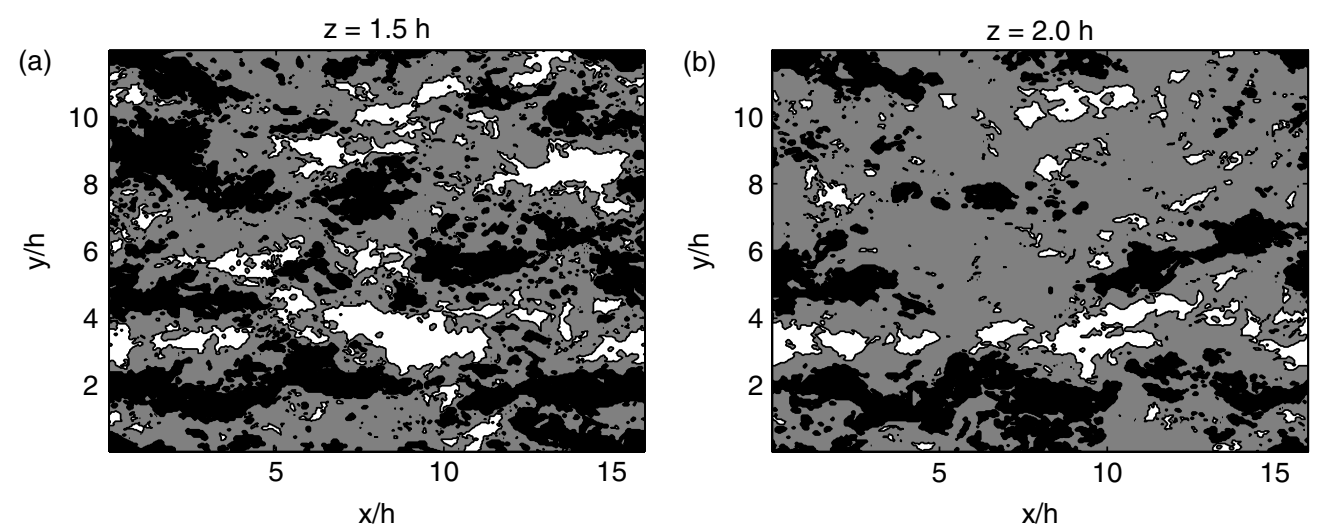

Figure 3. Flow visualization of low momentum regions (large-scale structures) in a horizontal snapshot above the buildings: (a) at $z=1.5 \mathrm{~h}$ (b) at $z=2.0 \mathrm{~h}$. Black regions: $u<0.8 \mathrm{u}_{\mathrm{m}}$. White regions: $u>1.2 u_{\mathrm{m}}$. Here $u_{\mathrm{m}}$ is the local mean velocity.
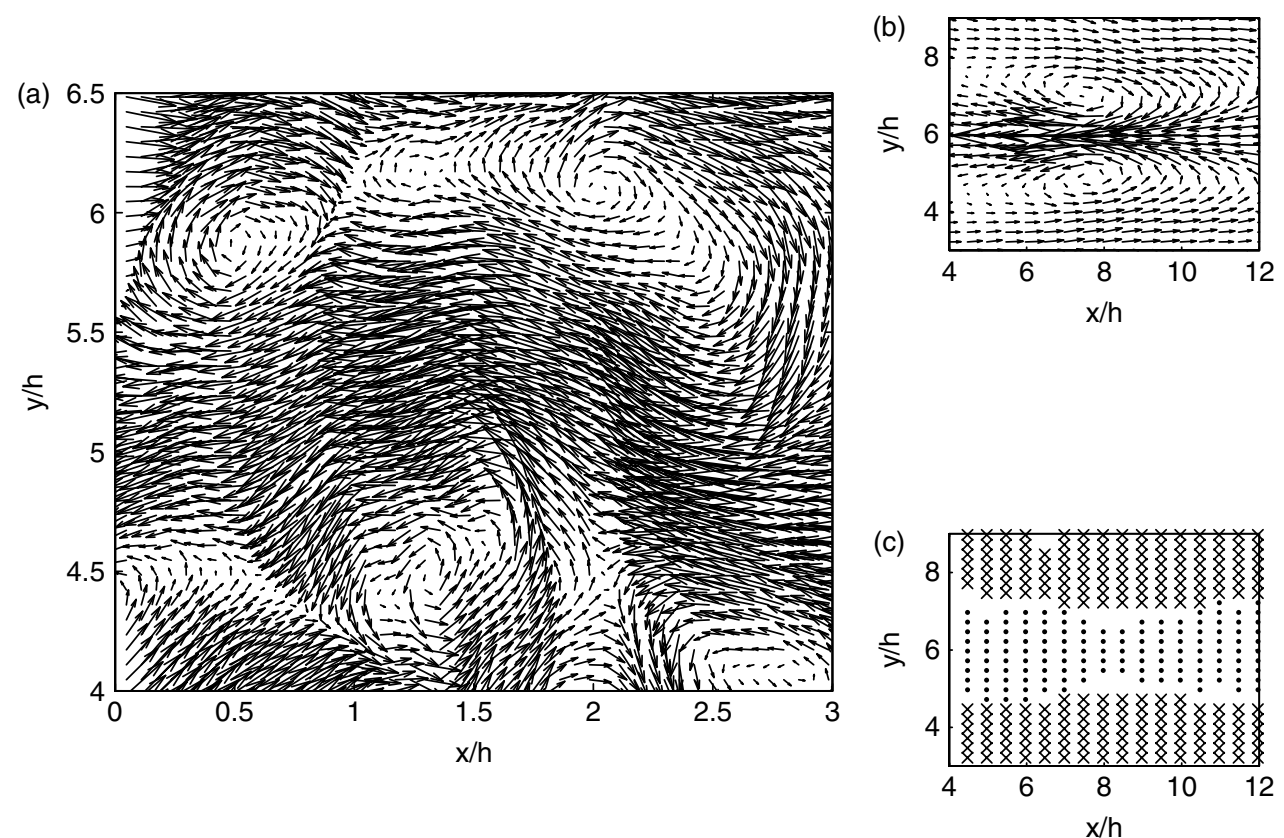

Figure 4. Flow visualization of hairpin vortex packets above canopy: (a) velocity vectors (u,v) in a snapshot in the $\mathrm{x}-\mathrm{y}$ plane at $z=1.5 \mathrm{~h}$ after Galilean decomposition and low pass filtering, (b) conditionally averaged structure in the same plane, (c) locations of ejection (dots) and sweep (crosses) events in that plane. Vectors and symbols are plotted at reduced resolution for clarity.

the streamwise direction - a hairpin vortex packet - that then induce an elongated region of low momentum that is much longer than the characteristic length scale of the individual hairpins.

We note that the mechanism outlined above depends only on the presence of an initial vortical disturbance or strong ejection event in a sheared mean flow ( $c f$. Suponitsky et al., 2005). Given the generality of this mechanism, it is therefore reasonable to expect hairpin vortex packets to be present in a wide variety of shear flows, including the present. Indeed there has been some experimental evidence for their presence over natural rough surfaces such as in the atmospheric boundary layer (Hommema and Adrian, 2002) and in the bottom boundary layer of coastal oceans (Smith et al., 2005).

The existence of hairpin vortex packets in the present flow may be revealed by instantaneous flow visualization using a technique of Galilean decomposition together with low pass filtering (Tomkins and Adrian, 2003). The resulting pattern of counter-rotating vortex cores, shown in Figure 4(a) in a horizontal plane at $z=1.5 h$, is very similar to those educed by Tomkins and Adrian (2003) over a smooth surface.

The vortex structures are analysed statistically by conditional averaging using the following procedure. First, a trigger event needs to be specified that can identify the location of the vortices. Following Tomkins and Adrian (2003), this trigger is here taken to be a local velocity minimum below a threshold set at $75 \%$ of the local mean velocity. This trigger is based on the fact that the hairpin vortex legs induce a reverse flow that gives an LMR with an associated velocity minimum. Secondly, the locations of points where the trigger event occurs are identified in a large number of independent snapshots. Thirdly, the 

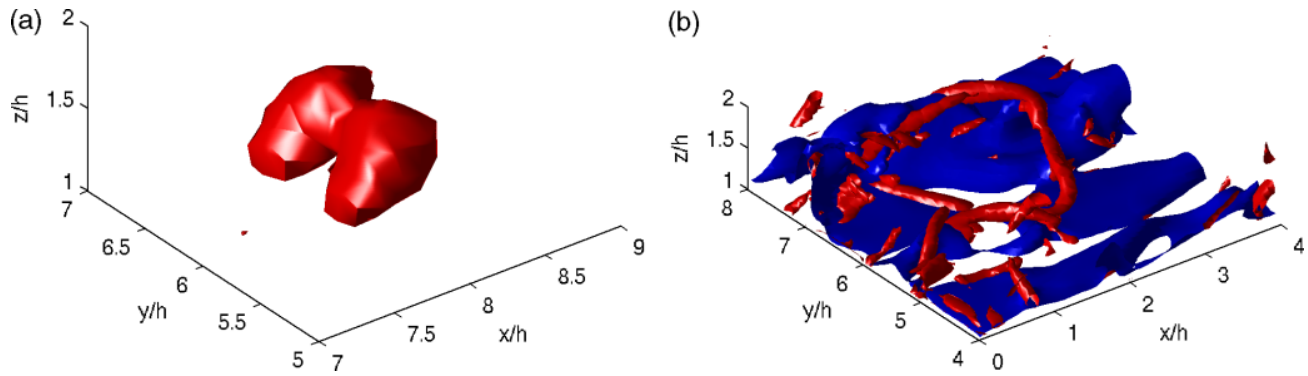

Figure 5. (a) 3D structure of the conditional eddy. (b) Instantaneous visualization of 3D structure at a lower Reynolds number of $R e^{\tau}=125$, showing low momentum regions (blue) and 'hairpin' and 'cane' vortices (red). Note that individual realizations of hairpin vortices are not usually symmetrical. The vortex cores are visualized using negative isosurfaces of $\lambda_{2}$ (Jeong and Hussain, 1995). This figure is available in colour online at www.interscience.wiley.com/ijoc
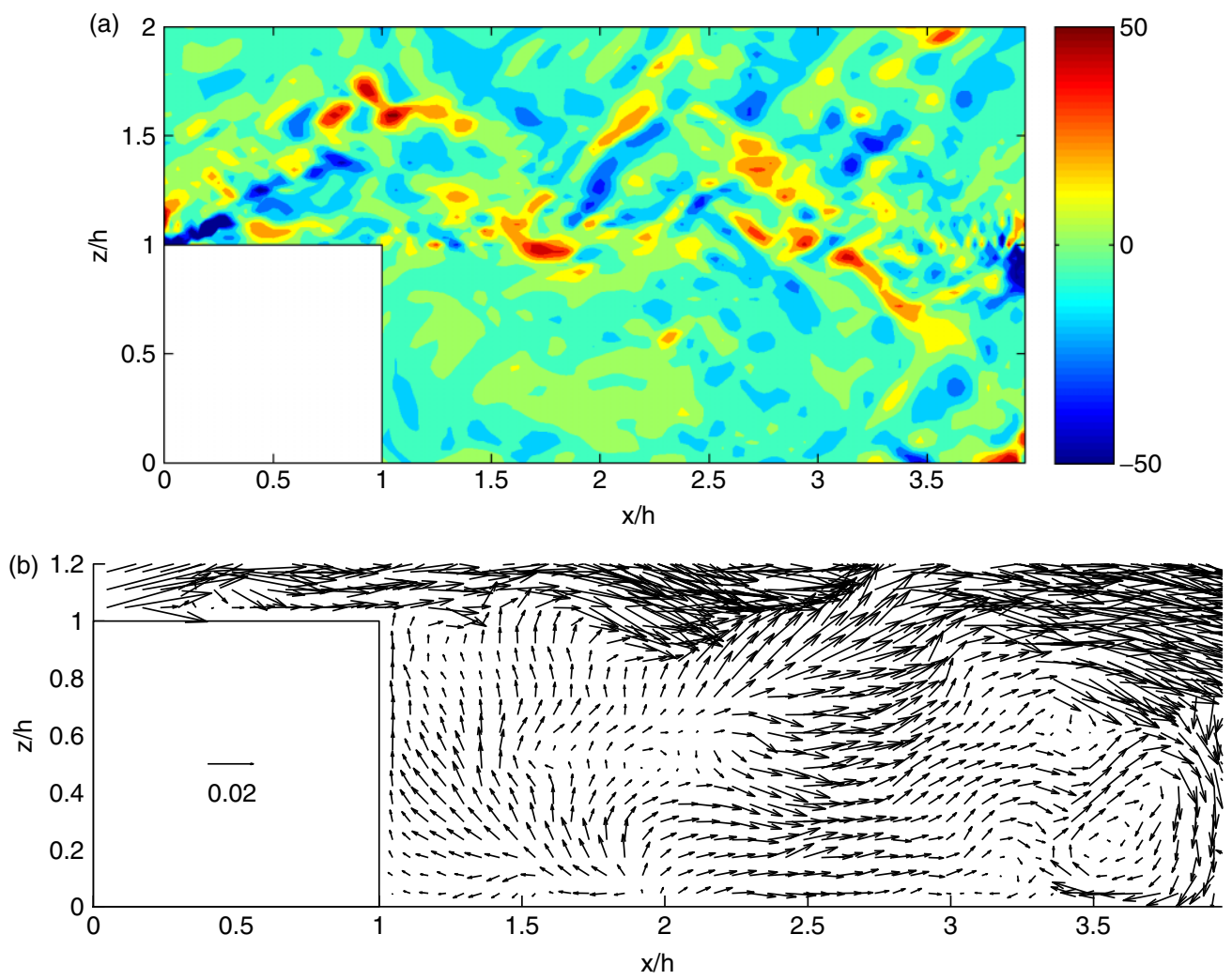

Figure 6. (a) Structure of the shear layer visualized by contours of spanwise vorticity in an x-z plane through the middle of a cube. Values of vorticity shown on colour bar are in non-dimensional units and are normalized by $u^{\tau} / h$. Here $u^{\tau}$ is the friction velocity based on the total stress and $h$ is the cube height. (b) Instantaneous wind vector plot in the same plane. Note the recirculation in front of the cube, caused by intermittent impinging of the shear layer. Vectors are plotted at reduced resolution for clarity. The inserted arrow indicates the scale of the velocity vectors, with the given value being velocity normalized by $\mathbf{u}^{\tau}$. This figure is available in colour online at www.interscience.wiley.com/ijoc

coordinates are shifted to centre on the trigger location in each realization, and the data is averaged over all realizations. Conditional averaging of a large number of realizations of these structures gives the 'conditional eddy' - a pair of counter-rotating vortices inducing a LMR in between (Figure 4(b)). Ejections are located in between the vortex legs and sweeps on the sides (Figure 4(c)). The 3D structure of the hairpin-like conditional eddy is shown in Figure 5(a), and has been visualized using negative isosurfaces of the $\lambda_{2}$ vortex identifier proposed by Jeong and Hussain (1995). The vortex cores visualized by the $\lambda_{2}$ method do not coincide exactly with the centres of circulation on the corresponding wind vector plots
(Jeong and Hussain, 1995). This explains why the conditional eddy visualized in Figure 5(a) appears smaller than in Figure 4(b). Actual realizations of these vortices may be visualized more easily at lower Reynolds numbers. Figure 5(b) shows an instantaneous snapshot with the vortices (red) visualized using negative $\lambda_{2}$ isosurfaces and low momentum regions (blue) visualized as isosurfaces of low velocity. The Reynolds number here is $R e_{\tau}=125$, or $R e^{*}=5.6$, which is still well above the threshold for fully rough flow (e.g. Snyder and Castro, 2002 cite $R e^{*}>1$ as the criterion for fully rough flow over arrays of sharp edged obstacles). Note the differences between the appearance of the conditional 
eddy and the actual realizations. In particular, the conditional hairpin appears much fatter because it is the smeared-out composite of many realizations of hairpins of different shapes and sizes. We also note that individual hairpins are usually not symmetrical, and may only have one leg ('cane' vortices); the symmetrical hairpin is an idealization.

The spanwise length scale of the conditional eddy may be measured and is found to increase linearly with height from about $z=1.5 h$ up to about $3 h$, which corresponds to the extent of the $\log$ region; this linear scaling of eddy sizes in the log region is a property of attached eddies as defined by Townsend (1976). Our results are consistent with those of Castro et al. (2006) who found that spanwise integral length scales obtained from twopoint correlations increased with height. Tomkins and Adrian (2003) also observed a linear scaling of eddy sizes in the corresponding log region over smooth walls, but the eddy sizes there were several times smaller than the present ones. Close to the canopy top, the spanwise length scale is of order $2 h$, and is much larger than the corresponding value for smooth wall hairpins near the wall. This is a reflection of the different generating mechanisms responsible for creating the primary vortices. In the present case, as explored in the next section, vortex shedding by the buildings seeds the flow with initial primary vortices, whereas a viscous mechanism operates in the case of smooth walls.

\section{The canopy-top shear layer}

Eddy structures formed by rolling-up of shear layers are shed at the top of individual buildings. This is visualized for a snapshot in Figure 6(a) by plotting contours of spanwise vorticity in a vertical $x-z$ plane through the middle of a building. When building heights are uniform as in the present case and consecutive buildings are relatively close together this gives a strong and persistent canopy-top shear layer. This shear layer can act in several ways. On the one hand it 'decouples' in a time mean sense the canopy from the flow above, leading to a blocking effect (Hunt and Durbin, 1999). This apparent decoupling, whereby interaction between the canopy flow and that above appears to be restricted to smaller scales, is reflected in a linear effective mixing length profile both above and within the canopy, with a minimum at the canopy top (Coceal et al., 2006). On the other hand the shear layer plays a crucial role in the intermittent coupling of the canopy flow with that above.

The flapping of the shear layers sheds eddy structures that alternately escape into the region aloft where they
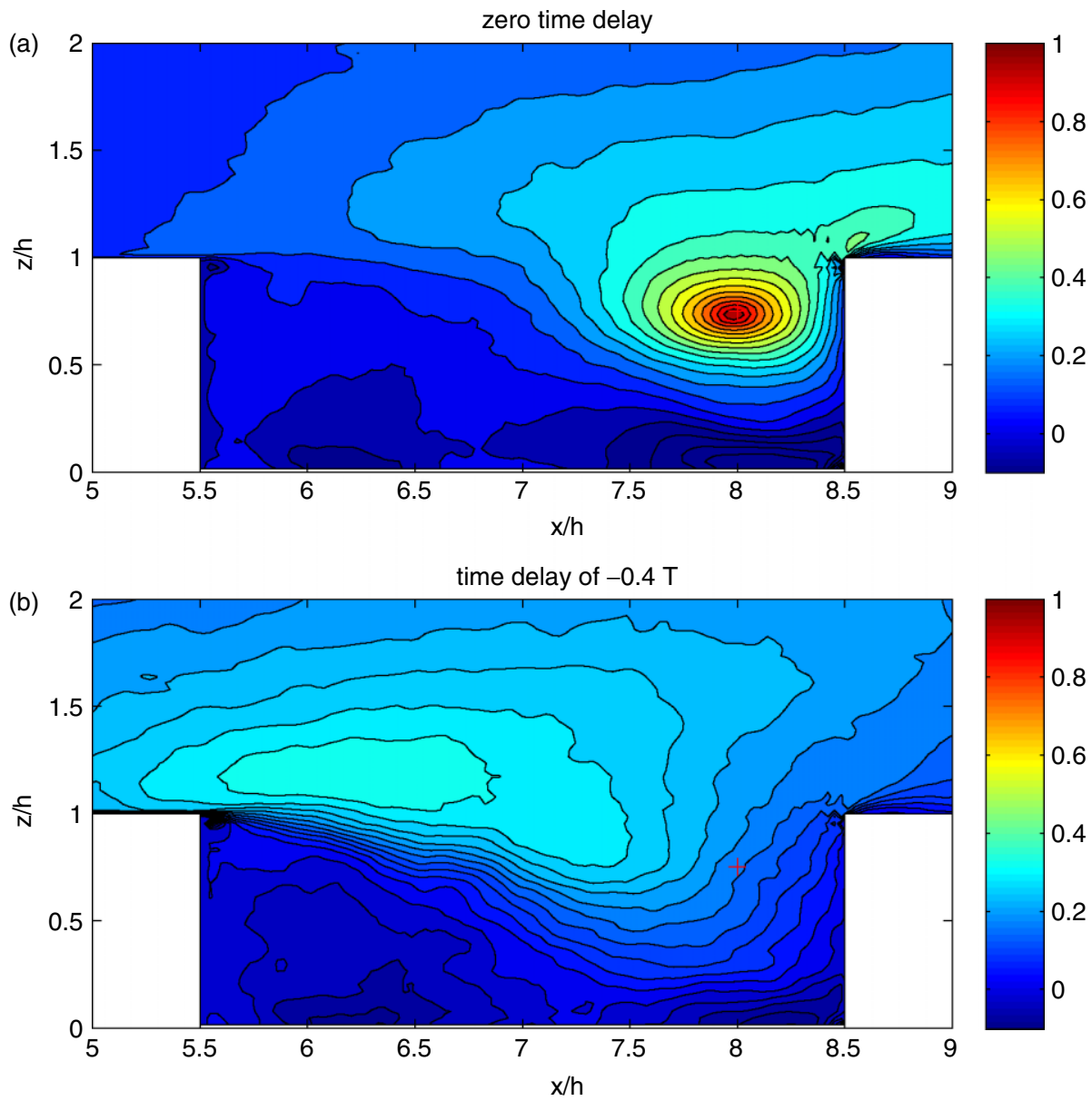

Figure 7. Two-point correlations $R_{u u}$ to show coupling of canopy flow with above flow: (a) Equal-time correlations with reference point at $(\mathrm{x}=8 \mathrm{~h}, \mathrm{z}=0.75 \mathrm{~h}$. (b) Correlations with negative time delay of $-0.4 T$. This figure is available in colour online at www.interscience.wiley.com/ijoc 
initiate hairpin vortex packets via the parent-offspring mechanism described in the last section; or penetrate down into the canopy where they may then impinge on downstream buildings, driving an intermittent recirculation in the canopy (Figure 6(b)). The latter scenario is consistent with the results of field measurements performed by Louka et al. (2000).

The coupling of the flow in the upper canopy with the shear layer structures upstream is confirmed statistically by computing space-time two-point velocity correlations $R_{\mathrm{uu}}$, defined by

$$
R_{\mathrm{uu}}(r, \delta r, \delta t)=\frac{\overline{u^{\prime}(r, t) u^{\prime}(r+\delta r, t+\delta t)}}{\sqrt{\overline{u^{\prime 2}(r, t)}} \sqrt{\overline{u^{\prime 2}(r+\delta r, t+\delta t)}}}
$$

where $r$ denotes the location $(\mathrm{x}, \mathrm{y})$ of the reference point, $\delta r$ is the separation between the points and $\delta t$ is the time delay. The reference point is taken to be at $z=0.75 h$ within the canopy and a distance $\mathrm{x}=0.5 h$ in front of a building. Figure 7(a) and (b) show contour plots of $R_{\text {uи }}$ without time delay and with a negative time delay of $-0.4 T$ respectively, where $T$ is an eddy turnover time of the largest eddies shed by the buildings. The latter plot shows significant correlation between the recirculation in front of the building and the shear layer structures above the upstream building at the earlier time.

The frequency of coupling between the shear layer structures and the canopy flow may be deduced from the Fourier transform of the cross correlation function $R_{\mathrm{uu}}$ between two points, with one located just above the buildings and one within the canopy. This cross-spectrum (not shown) indicates that the coupling takes place at frequencies between $0.1 / T$ and $1 / T$ (i.e. on time scales between $T$ and $10 T$ ) that correspond to the peak in the cross-spectrum function. This peak is very broad, an indication that the coupling between the shear layer and the flow within the canopy has multi-scale characteristics. This is not surprising due to the complexity of the highly three-dimensional flow at this interface along with its intermittent characteristics.

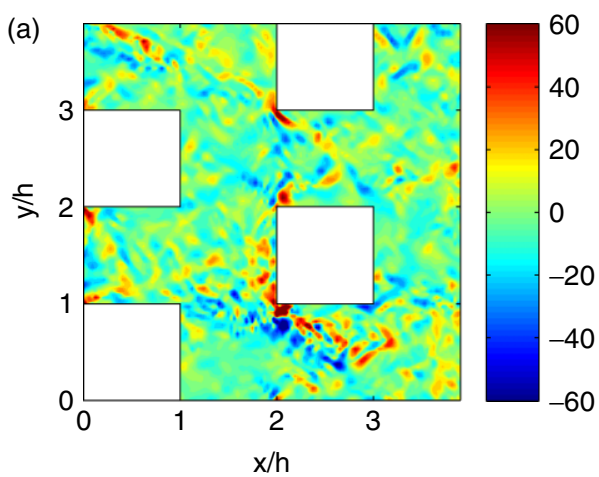

\section{Turbulent structures within the canopy layer}

In addition to the intermittent recirculation in front of the buildings, the coupling with the shear layer and the boundary layer structures leads to intermittent but strong sweep and ejection events. Examples of such events are depicted in the vector plots of Figure 8. These violent episodes are potentially important in the venting and dispersion of pollutants as well as contributing a majority of the vertical transport of momentum.

Within the canopy there are also eddies shed off the vertical faces of the buildings. To visualize these,
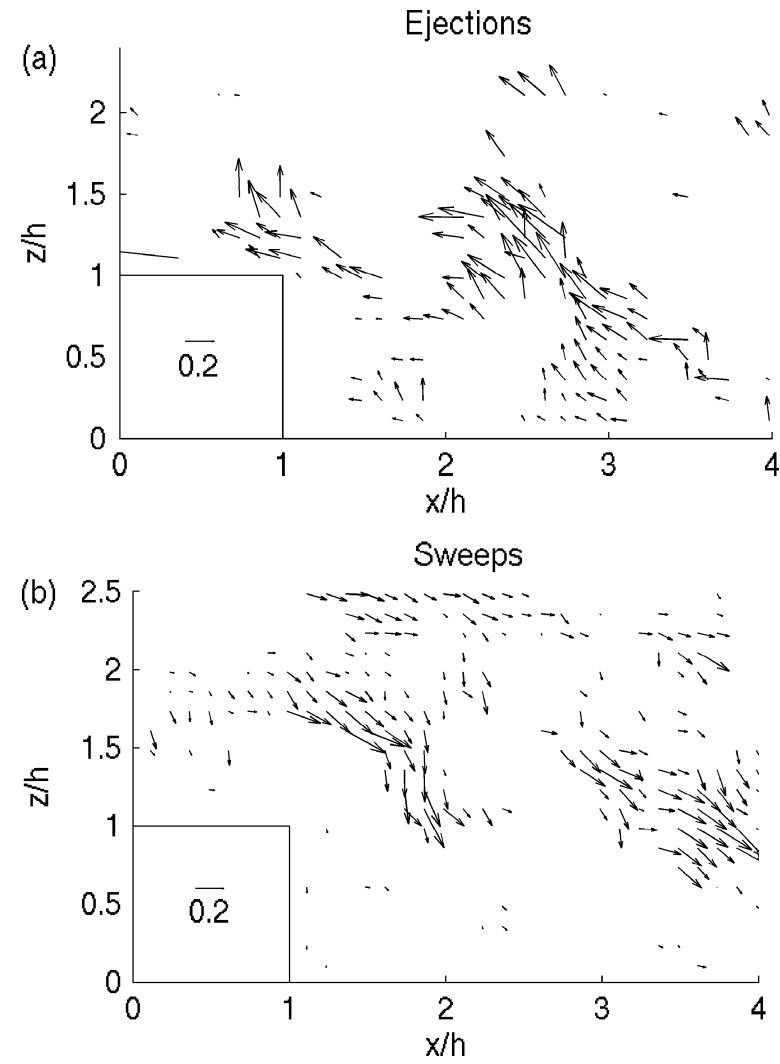

Figure 8. Snapshot of wind vectors in an $\mathrm{x}-\mathrm{z}$ plane through the middle of a cube showing: (a) ejections (b) sweeps. Vectors are plotted at greatly reduced resolution for clarity. The inserted arrow indicates the scale of the velocity vectors, with the given value being velocity normalized by $\mathbf{u}^{\tau}$.

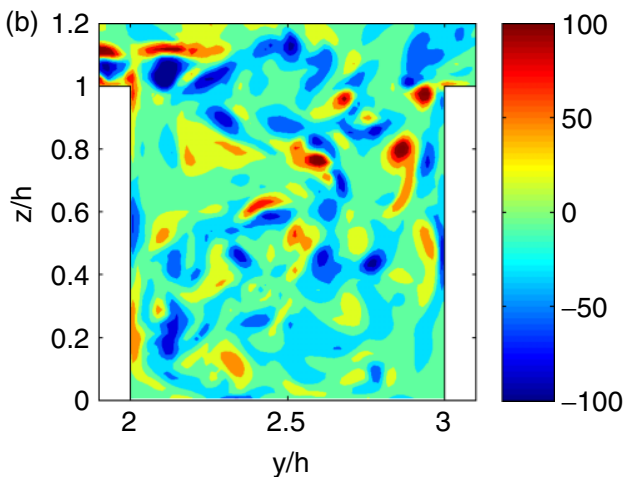

Figure 9. (a) Visualization of vertical shear layers within the canopy by contours of vertical vorticity in an $x-y$ plane at $z=0.5 h$. (b) Streamwise vorticity within the canopy in a y-z plane through the middle of the cubes shown. Values of vorticity shown on colour bar are in non-dimensional units and are normalized by $\mathrm{u}^{\tau} / h$. This figure is available in colour online at www.interscience.wiley.com/ijoc 
Figure 9(a) shows contour plots of vertical component of vorticity in a horizontal $\mathrm{x}-\mathrm{y}$ plane at $z=0.5 \mathrm{~h}$ in part of the domain. These eddies interact with each other and that interaction leads to enhanced lateral mixing.

The presence of numerous streamwise eddies is also apparent in the $y-z$ plane by inspection of wind vector and vorticity plots. Figure 9(b) shows an example, where contours of streamwise vorticity reveal many vortical structures within the canopy with opposite sense of rotation. These quasi-streamwise structures are most probably caused by the tilting and stretching by the mean shear of the vertical vorticity associated with the vertical eddies.

Over sufficiently long timescales the mean flow structure around the buildings also becomes important. The mean flow is associated with significant dispersive stresses within the building canopy (Coceal et al., 2006), and gives rise to topological dispersion (Belcher, 2005). Unfortunately, the mean flow structure is very dependent on the geometry and layout of the building array, as shown by Coceal et al. (2006).

In conclusion, the flow dynamics within the building canopy is much more complicated than that above, due to interaction between several processes. One might reasonably expect that details within the building canopy affect the scale of turbulent structures but not the nature of the dynamics above the canopy. However, the flow structures within the canopy, their scales and interactions are likely to depend much more on the local geometry. For this reason it remains a challenge to characterize the flow within the building canopy with any generality.

\section{CONCLUSIONS AND DISCUSSION}

It is shown in this paper that DNS performed over an idealized urban canopy are able to reproduce turbulence statistics accurately, and also to obtain detailed information about the structure and dynamics of turbulence. Here, we emphasize the need to evaluate such numerical studies carefully. Evaluation involves two separate aspects. The first is basically a check of the accuracy of the numerical simulation. For this purpose it is desirable to make quantitative comparisons with a controlled experiment that is as close as possible in configuration to the numerical simulation. This is almost impossible to achieve in field experiments, but is readily done in the wind tunnel. A second aspect of model evaluation is to check if the simulation captures features and processes observed in real atmospheric data. Given the difficulties of matching the urban setup and flow conditions, the best that can usually be done in practice is a more qualitative comparison. The present simulations have been evaluated mainly in the first sense by the high level of agreement with wind tunnel data, but we also note the qualitative correspondence between some findings in this paper and observations in the real atmosphere (e.g. evidence of hairpin vortices observed in the atmospheric boundary layer by Hommema and Adrian (2002) and intermittent impinging of the shear layer on downstream buildings observed by Louka et al. (2000)). It is also hoped that a proper quantitative evaluation of the present numerical results against real atmospheric data for a matching urban configuration may be performed in future.

On the basis of the findings in this paper, the unsteady turbulent dynamics over a group of buildings may be summarized in the cartoon shown in Figure 10. Three regimes of the flow may be identified. First, well above

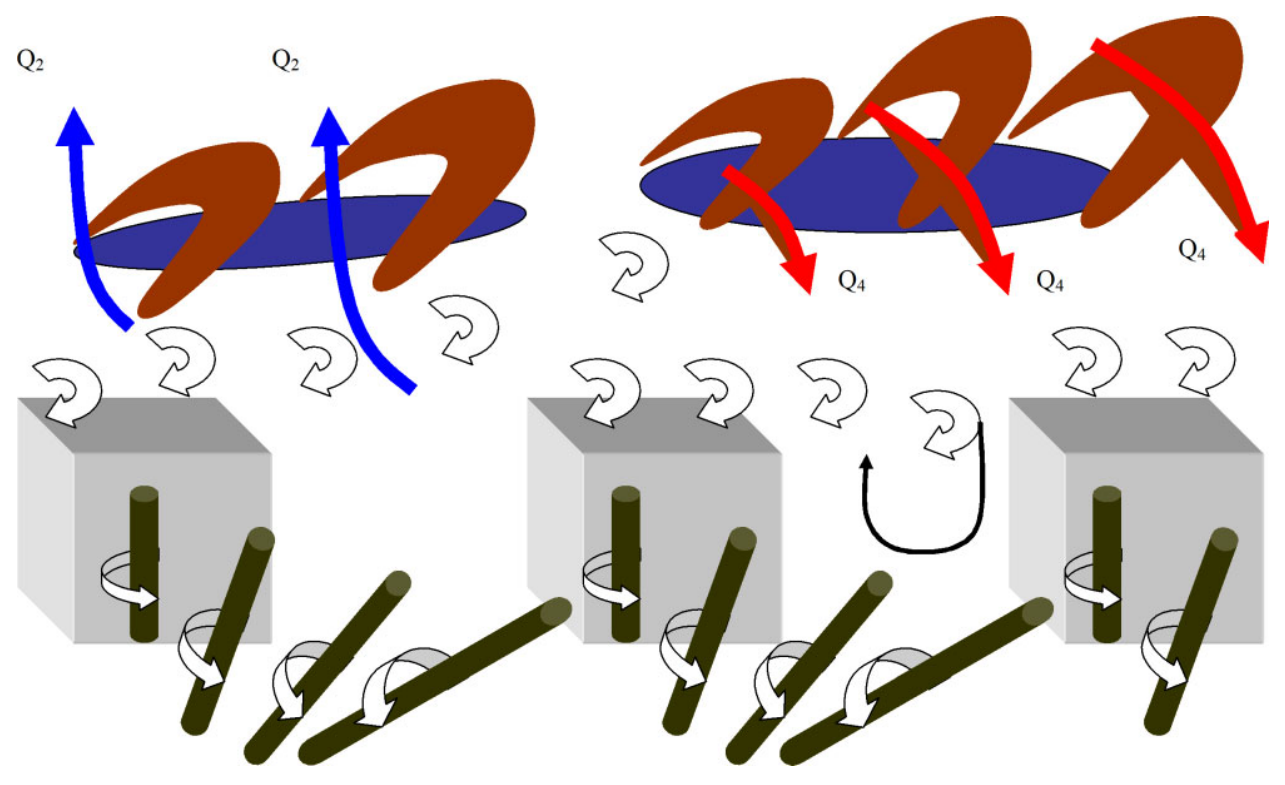

Figure 10. Conceptual cartoon summarising the unsteady flow dynamics above and within groups of buildings. Above the buildings momentum transport is dominated by large hairpin-shaped eddies (brown) with associated low momentum regions (blue). $\mathrm{Q}_{2}$ denotes an ejection event between the hairpin vortex legs and $\mathrm{Q}_{4}$ denotes a sweep event on the sides. Within the building canopy eddies shed off the vertical edges of the buildings are rotated by the mean shear. Strong canopy-top shear layers intermittently penetrate into the canopy, impacting upon buildings downstream and driving a recirculation in front of the buildings. This figure is available in colour online at www.interscience.wiley.com/ijoc 
the buildings there is a rough wall boundary layer. We have shown that the dominant organized structures in that region in fact resemble those over a smooth wall boundary layer, so that transport of momentum and presumably scalars would be dominated by the hairpin vortices, with associated low momentum regions. We speculate that these low momentum regions would presumably contain high scalar concentrations, although this is as yet an unverified conjecture. Our results confirm and extend the visualization studies of these structures by Kanda et al. (2004) and Kanda (2006), by performing conditional averaging of the coherent structures to establish their statistical dominance, association and length scales. Moreover, the conditional eddy as well as its instantaneous realizations have been visualized in $3 \mathrm{D}$, and are shown to have a hairpin-like shape. The high resolution used in the present DNS also allows one to study the shedding of eddies by the buildings and smaller scale structures within the building canopy. It is found that close to the canopy top strong shear layer structures shed off the tops of buildings become important, and they interact both with the boundary layer eddies and the canopy flow. These shear layer structures impinge intermittently upon any downstream buildings that may be present and drive a recirculation in front of the buildings. Within the building canopy, the flow dynamics is complicated by a complex interaction between eddies shed off the vertical faces of buildings, the intermittent penetration of the canopy by the shear layer structures at the canopy top and larger boundary layer eddies, and the result of these vortices impacting upon buildings. Additionally, the vertical vorticity is tilted and stretched by the mean shear and gives rise to inclined vortices within the canopy.

Note the similarities, but also the differences compared to the phenomenological model described by Poggi et al. (2004) in the case of vegetation canopies. Well above the roughness both flows must resemble each other at least qualitatively since both are rough wall flows ( $c f$. Raupach et al., 1991). However, the corresponding pictures in the roughness sublayer and canopy layer are very different. In a vegetation canopy the inflection in the velocity profile near the canopy top gives rise to an inviscid instability that generates mixing-layer type vortices (Raupach et al., 1996; Finnigan, 2000). These eddies have a length scale that is related to the spatially averaged profile and is thus of canopy scale. For dense canopies, they are the most dominant organized structures. In an urban canopy, although the spatially-averaged mean velocity profile also has a marked inflection at the canopy top, it is not dynamically significant, being just an artefact of the spatial averaging process. Hence, there is no generation of large canopy-scale eddies. Because the buildings are large in relation to the canopy scale (as opposed to vegetation), eddy structures shed off the individual buildings are more important. Well within the depth of a vegetation canopy, Poggi et al. (2004) argued that the dominant coherent motions are von Karman vortex streets of a single length scale proportional to the diameter of the trees, whereas we have seen in the present results that the flow within a building canopy is the result of a complicated interaction between different coherent motions. In this case, it does not appear that a single length scale predominates.

It is not claimed that the conceptual model outlined in Figure 10 is the complete picture, and much further work is needed to fill in the many gaps in our understanding. These include, but are not restricted to, the following points:

- The need to quantify the scales of the turbulent structures in the three aforementioned regimes.

- A more detailed study of the structures within the building canopy.

- The need to quantify the importance of the unsteady organized motions vs. the mean flow on transport of momentum and scalars.

- How the coupling of the flow in the building canopy to the flow above is modified in more realistic building geometries, including variable building heights.

\section{ACKNOWLEDGEMENTS}

O.C. would like to extend his thanks to the following: Ian Castro and Hong Cheng for kind permission to use their experimental data for comparison; Stephen Belcher and John Finnigan for useful discussions; NCAS/NERC for funding.

\section{REFERENCES}

Adrian RJ, Meinhart CD, Tomkins CD. 2000. Vortex organization in the outer region of the turbulent boundary layer. Journal of Fluid Mechanics 422: 1-54.

Belcher SE. 2005. Mixing and transport in urban areas. Philosophical Transactions of the Royal Society 363: 2947-2963.

Britter RE, Hanna SR. 2003. Flow and dispersion in urban areas. Annual Review of Fluid Mechanics 35: 469-496.

Castro IP, Cheng H, Reynolds R. 2006. Turbulence over urban-type roughness: deductions from wind tunnel measurements. BoundaryLayer Meteorology 118: 109-131.

Cheng H, Castro IP. 2002. Near wall flow over urban-like roughness. Boundary-Layer Meteorology 104: 229-259.

Christen A. 2005. Atmospheric turbulence and surface energy exchange in urban environments. $\mathrm{PhD}$ thesis, Basel.

Coceal O, Belcher SE. 2004. A canopy model of mean winds through urban areas. Quarterly Journal of the Royal Meteorological Society 130: $1349-1372$.

Coceal O, Belcher SE. 2005. Mean winds through an inhomogeneous urban canopy. Boundary-Layer Meteorology 115: 47-68.

Coceal O, Thomas TG, Castro IP, Belcher SE. 2006. Mean flow and turbulence statistics over groups of urban-like cubical obstacles. Boundary-Layer Meteorology 121: 491-519.

Feigenwinter C, Vogt R. 2005. Detection and analysis of coherent structures in urban turbulence. Theoretical and Applied Climatology 81: $219-230$.

Finnigan JJ. 2000. Turbulence in plant canopies. Annual Review of Fluid Mechanics 32: 519-572.

Grass AJ. 1971. Structural features of turbulent flow over smooth and rough boundaries. Journal of Fluid Mechanics 50: 233-255.

Grimmond CSB, Oke TR. 1999. Aerodynamic properties of urban areas derived from analysis of surface form. Journal of Applied Meteorology 38: 1262-1292.

Hommema SE, Adrian RJ. 2002. Packet structure of surface eddies in the atmospheric boundary layer. Boundary-Layer Meteorology 106: $147-170$.

Hunt JCR, Durbin PA. 1999. Perturbed vortical layers and shear sheltering. Fluid Dynamics Research 24: 375-404.

Jeong J, Hussain F. 1995. On the identification of a vortex. Journal of Fluid Mechanics 285: 69-94. 
Jimenez J. 2004. Turbulent flows over rough walls. Annual Review of Fluid Mechanics 36: 173-196.

Kanda M. 2006. Large-eddy simulations on the effects of surface geometry of building arrays on turbulent organized structures. Boundary-Layer Meteorology 118: 151-168.

Kanda M, Moriwaki R, Kasamatsu F. 2004. Large-eddy simulation of turbulent organized structures within and above explicitly resolved cube arrays. Boundary-Layer Meteorology 112: 343-368.

Louka P, Belcher SE, Harrison RG. 2000. Coupling between the air flow in streets and the well-developed boundary layer aloft. Atmospheric Environment 34: 2613-2621.

Oikawa S, Meng Y. 1995. Turbulence characteristics and organized motion in a suburban roughness sublayer. Boundary-Layer Meteorology 74: 289-312.

Poggi D, Porporato A, Ridolfi L, Albertson JD, Katul GG. 2004 The effect of vegetation density on canopy sub-layer turbulence. Boundary-Layer Meteorology 111: 565-587.

Raupach MR, Antonia RA, Rajagopalan S. 1991. Rough-wall turbulent boundary layers. Applied Mechanics Reviews 44: 1-25.

Raupach MR, Finnigan JJ, Brunet Y. 1996. Coherent eddies and turbulence in vegetation canopies: the mixing layer analogy. Boundary-Layer Meteorology 78: 351-382.

Roth M. 2000. Review of atmospheric turbulence over cities. Quarterly Journal of the Royal Meteorological Society 126: 941-990.
Smith WAMN, Katz J, Osborn TR. 2005. On the structure of turbulence in the bottom boundary layer of the coastal ocean. Journal of Physical Oceanography 35: 72-93.

Snyder WH, Castro IP. 2002. The critical Reynolds number for roughwall boundary layers. Journal of Wind Engineering and Industrial Aerodynamics 90: 41-54.

Suponitsky V, Cohen J, Bar-Yoseph PZ. 2005. The generation of streaks and hairpin vortices from a localized vortex disturbance embedded in unbounded uniform shear flow. Journal of Fluid Mechanics 535: 65-100.

Tomkins CD, Adrian RJ. 2003. Spanwise structure and scale growth in turbulent boundary layers. Journal of Fluid Mechanics 490: 37-74. Townsend AA. 1976. The Structure of Turbulent Shear Flow. Cambridge University Press, Cambridge.

Xie Z, Castro IP. 2006. LES and RANS for turbulent flow over arrays of wall-mounted obstacles. Flow Turbulence and Combustion 76: 291-312.

Yao YF, Thomas TG, Sandham ND, Williams JJR. 2001. Direct numerical simulation of turbulent flow over a rectangular trailing edge. Theoretical and Computational Fluid Dynamics 14: 337-358. Zhou J, Adrian RJ, Balachandar S, Kendall TM. 1999. Mechanisms for generating coherent packets of hairpin vortices in channel flow. Journal of Fluid Mechanics 387: 353-396. 